\title{
Vögel und Frösche
}

\author{
Freeman Dyson
}

Manche Mathematiker sind Vögel, andere sind Frösche. Die Vögel fliegen hoch oben in der Luft und überblicken weite Bereiche der Mathematik bis zum fernen Horizont. Sie haben Freude an Ideen, die unser Denken vereinheitlichen und unterschiedliche Probleme aus den verschiedenen Regionen dieser Landschaft zusammenführen. Frösche leben unten im Schlamm und sehen nur die Blumen, die in der Nähe wachsen. Sie haben Freude an den Details von Dingen und lösen immer nur ein Problem nach dem anderen. Ich bin zufällig ein Frosch, aber viele meiner besten Freunde sind Vögel. Und um das wird es in meinem Vortrag heute Abend vor allem gehen. Die Mathematik braucht sowohl Vögel als auch Frösche. Sie ist deshalb so vielfältig und schön, weil Vögel das große Ganze im Blick behalten und Frösche komplizierteste Details liefern. Weil sie die Allgemeinheit von Begriffen mit der Tiefe von Systemen verbindet, ist die Mathematik zugleich große Kunst und bedeutende Wissenschaft. Es wäre töricht zu behaupten, Vögel seien besser als Frösche, weil sie über größeren Weitblick verfügen, oder Frösche seien besser als Vögel, weil sie einen tieferen Einblick haben. Die Welt der Mathematik ist sowohl weit als auch tief, und wir brauchen Vögel und Frösche, die gemeinsam an ihrer Erforschung arbeiten.

Dieser Vortrag heißt Einstein-Vorlesung, und ich danke der American Mathematical Society, dass sie mich eingeladen hat, Albert Einstein die Ehre zu erweisen. Einstein war kein Mathematiker, sondern Physiker und stand der Mathematik mit gemischten Gefühlen gegenüber. Einerseits hatte er enormen Respekt vor der Fähigkeit der Mathematik, die Abläufe in der Natur zu beschreiben, und ein Gespür für mathematische Schönheit, das ihn bei der Suche nach Naturgesetzen auf den richtigen Weg brachte. Andererseits interessierte er sich nicht für reine Mathematik und hatte keinerlei technische Fertigkeiten als Mathematiker. In späteren Jahren stellte er jüngere Kollegen als Assistenten ein, damit sie mathematische Berechnungen für ihn durchführten. Seine eigene Denkweise war eher physikalisch als mathematisch. Unter Physikern nahm er als ein Vogel, der weiter als andere sah, eine herausragende Stellung ein. Ich werde jedoch nicht über Einstein sprechen, weil ich zu ihm nichts Neues zu sagen habe.

\section{Francis Bacon und René Descartes}

$\mathrm{Zu}$ Beginn des siebzehnten Jahrhunderts verkündeten zwei große Philosophen, Francis Bacon in England und René Descartes in Frankreich, die Geburt der modernen Wissenschaft. Descartes war ein Vogel, und Bacon war ein Frosch. Beide beschrieben ihre Vorstellung von der Zukunft, und diese Vorstellungen waren sehr unterschiedlich. Bacon sagte: „Es kommt alles darauf an, dass man stets die Tatsachen der Natur im Blick behält.“ Descartes sagte: „Ich denke, also bin ich." Bacon zufolge sollten Wissenschaftler durch die Welt reisen und Fakten sammeln, bis die zusammengetragenen Fakten erkennen lassen würden, wie die Natur funktioniert. Die Wissenschaftler könnten dann aus den Fakten die Gesetze ableiten, denen die Natur gehorcht. Descartes zufolge sollten die Wissenschaftler zu Hause bleiben und die Naturgesetze durch reines Denken erschließen. Um die Gesetze richtig herzuleiten, bräuchten die Wissenschaftler nur die Regeln der Logik und das Wissen um die Existenz Gottes. Seit Bacon und Descartes vor vierhundert Jahren die Richtung gewiesen haben, ist die Wissenschaft rasant fortgeschritten, indem sie beide Wege gleichzeitig verfolgte. Weder der baconianische Empirismus noch der cartesianische Dogmatismus sind in der Lage, die Geheimnisse der Natur im Alleingang zu ergründen, aber beide zusammen waren erstaunlich erfolgreich. Englische Wissenschaftler sind seit vierhundert Jahren eher Baconianer und französische eher Cartesianer. Faraday, Darwin und Rutherford waren Baconianer; Pascal, Laplace und Poincaré Cartesianer. Für die Wissenschaft war die wechselseitige Befruchtung dieser zwei gegensätzlichen Kulturen eine große Bereiche- 


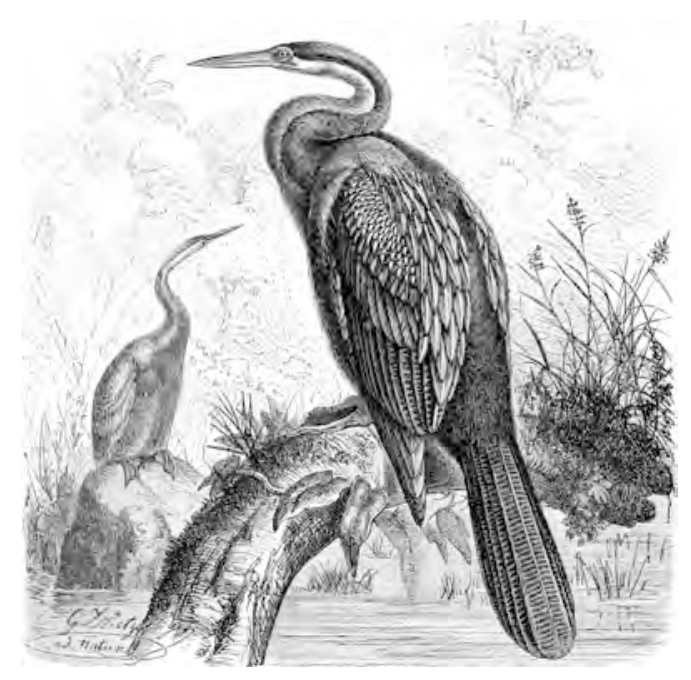

rung. Dabei waren beide Richtungen in jedem der Länder immer vertreten. Newton war im Grunde genommen ein Cartesianer, der das reine Denken im Sinne Descartes' benutzte, um das cartesianische Wirbeldogma zu widerlegen. Marie Curie war im Grunde genommen eine Baconianerin, die Tonnen von rohem Uranerz kochte, um das Dogma von der Unzerstörbarkeit der Atome niederzureißen.

In der Geschichte der Mathematik des zwanzigsten Jahrhunderts gab es zwei entscheidende Ereignisse, von denen eines in der baconianischen und das andere in der cartesianischen Tradition stand. Das erste war der Internationale Mathematikerkongress in Paris im Jahr 1900, auf dem Hilbert einen Grundsatzvortrag hielt, in dem er anhand seiner berühmten Liste von dreiundzwanzig ungelösten Problemen den Kurs der Mathematik im kommenden Jahrhundert skizzierte. Hilbert selbst war ein Vogel, der weit über dem gesamten Gebiet der Mathematik schwebte, aber er wandte sich mit seinen Problemen an die Frösche, die sie eines nach dem anderen lösen sollten. Das zweite entscheidende Ereignis war die Gründung der Bourbaki-Gruppe in den 193oer-Jahren in Frankreich, einer Gruppe mathematischer Vögel, die sich mit der Veröffentlichung einer Reihe von Lehrbüchern beschäftigte, um damit einen vereinheitlichten Rahmen für die gesamte Mathematik zu schaffen. Die Hilbert-Probleme haben die mathematische Forschung sehr erfolgreich in fruchtbare Bahnen gelenkt. Einige von ihnen wurden inzwischen gelöst, einige sind nach wie vor ungelöst, aber fast alle stimulierten die Entwicklung neuer Ideen und neuer Gebiete der Mathematik. Das Bourbaki-Projekt war ebenso einflussreich. Es veränderte in den folgenden fünfzig Jahren den Stil der Mathematik, indem es eine bis dahin nicht existierende logische Kohärenz durchsetzte und das Hauptaugenmerk von konkreten Beispielen auf abstrakte allgemeine Prinzipien verlagerte. Im Bourbaki-Verständnis ist Mathematik das in den BourbakiLehrbüchern enthaltene abstrakte System. Was nicht in den Lehrbüchern steht, ist keine Mathematik. Konkrete Beispiele sind keine Mathematik, weil sie in den Lehrbüchern nicht vorkommen. Das Bourbaki-Programm war der extreme Ausdruck der cartesianischen Denkweise. Es begrenzte das Gebiet der Mathematik, indem es die schönen Blumen ausschloss, die baconianische Reisende am Wegesrand sammeln würden.

Scherze der Natur

Für mich als Baconianer fehlt im Bourbaki-Programm vor allem das Überraschungsmoment. Das Bourbaki-Programm versuchte, die Mathematik logisch zu machen, aber wenn ich die Geschichte der Mathematik betrachte, sehe ich eine Abfolge von unlogischen Sprüngen, unwahrscheinlichen Zufällen, Scherzen der Natur. Einer der tiefgründigsten dieser Scherze ist die Quadratwurzel aus minus eins, die der Physiker Erwin Schrödinger in seine Wellengleichung einsetzte, als er im Jahr 1926 die Wellenmechanik erfand. Schrödinger war ein Vogel, der von der Idee einer Vereinigung von Mechanik und Optik ausging. Hundert Jahre zuvor hatte Hamilton die klassische Mechanik mit der Strahlenoptik in Einklang gebracht und zur Beschreibung von optischen Strahlen und klassischen Teilchenbahnen dieselbe Mathematik verwendet. Schrödingers Idee war es, diese Vereinheitlichung auf die Wellenoptik und Wellenmechanik auszudehnen. Die Wellenoptik gab es bereits, die Wellenmechanik jedoch noch nicht. Schrödinger musste die Wellenmechanik erst erfinden, um die Vereinigung zu bewerkstelligen. Ausgehend von dem Modell der Wellenoptik stellte er eine Differentialgleichung für ein mechanisches Teilchen auf, doch die Gleichung ergab keinen Sinn. Sie sah aus wie die Gleichung der Wärmeleitung in einem kontinuierlichen Medium. Die Wärmeleitung hat jedoch keine erkennbare Relevanz für die Teilchenmechanik. Schrödingers Idee schien damit ins Leere zu laufen. Doch dann folgte die Überraschung. Schrödinger setzte die Quadratwurzel aus minus eins in die Gleichung ein, und auf einmal ergab sie einen Sinn. Plötzlich wurde sie zu einer Wellengleichung statt zu einer Wärmeleitungsgleichung. Und Schrödinger fand zu seiner großen Freude heraus, dass es für die Gleichung Lösungen gibt, die den quantisierten Bahnen im Bohr'schen Atommodell entsprechen. 


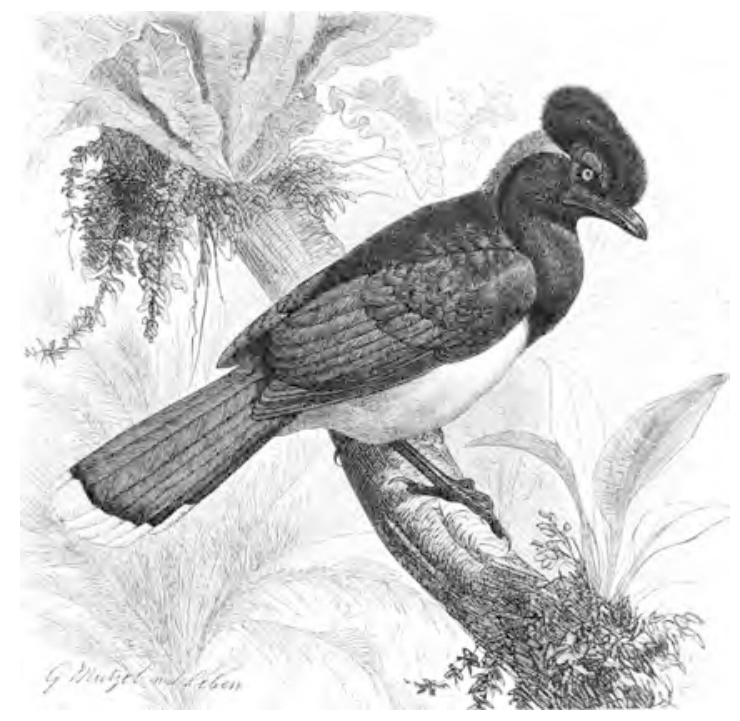

Es stellt sich heraus, dass die Schrödinger-Gleichung alles, was wir über das Verhalten von Atomen wissen, korrekt beschreibt. Sie ist die Grundlage der gesamten Chemie und größtenteils auch der Physik. Und die Quadratwurzel aus minus eins bedeutet, dass die Natur mit komplexen Zahlen und nicht mit reellen Zahlen operiert. Diese Entdeckung war für Schrödinger wie für jeden anderen eine völlige Überraschung. Wie Schrödinger später erzählte, sagte seine vierzehnjährige Freundin Itha Junger damals zu ihm: „Hey, als du damit angefangen hast, hast du noch nicht einmal daran gedacht, dass so viel Vernünftiges dabei herauskommen würde." Im neunzehnten Jahrhundert hatten Mathematiker von Abel bis Riemann und Weierstraß eine großartige Theorie der Funktionen komplexer Variablen entwickelt. Sie hatten entdeckt, dass die Funktionentheorie weitaus tiefgreifender und wirkungsvoller wurde, wenn sie von reellen auf komplexe Zahlen ausgedehnt wurde. Doch sie verstanden komplexe Zahlen stets als eine Konstruktion, die sich Mathematiker als nützliche und elegante Abstraktion von der Realität ausgedacht hatten. Ihnen ist nie in den Sinn gekommen, dass dieses von ihnen entworfene künstliche Zahlensystem tatsächlich die Grundlage der Bewegung von Atomen ist. Sie konnten sich einfach nicht vorstellen, dass die Natur zuerst darauf gekommen ist.

Ein weiterer Scherz der Natur ist die exakte Linearität der Quantenmechanik, die Tatsache, dass die möglichen Zustände eines jeden physikalischen Objekts einen linearen Raum bilden. Bevor die Quantenmechanik erfunden wurde, war die klassische Physik stets nichtlinear, und lineare Modelle waren nur näherungsweise gültig. Nach Entwicklung der Quantenmechanik wurde auf einmal die Natur selbst linear. Dies hatte tiefgreifende Konsequenzen für die Mathematik. Im neunzehnten Jahrhundert entwickelte Sophus Lie seine ausgeklügelte Theorie der kontinuierlichen Gruppen, die das Verhalten klassischer dynamischer Systeme erklären sollte. Lie-Gruppen waren damals weder für Mathematiker noch für Physiker von besonderem Interesse. Den Mathematikern war die nichtlineare Theorie der Lie-Gruppen zu kompliziert, und für die Physiker war sie zu unbedeutend.
Lie starb als enttäuschter Mann. Und dann stellte sich fünfzig Jahre später heraus, dass die Natur exakt linear und die Theorie der linearen Abbildungen von Lie-Algebren die natürliche Sprache der Teilchenphysik war. Lie-Gruppen und Lie-Algebren erlebten eine Wiedergeburt als eines der zentralen Themen in der Mathematik des zwanzigsten Jahrhunderts.

Ein dritter Scherz der Natur ist die Existenz von Quasikristallen. Im neunzehnten Jahrhundert führte die Erforschung von Kristallen zu einer vollständigen Liste möglicher diskreter Symmetriegruppen im euklidischen Raum. Theoreme wurden überprüft und die Tatsache nachgewiesen, dass diskrete Symmetriegruppen im dreidimensionalen Raum nur Drehungen der Ordnung drei, vier oder sechs enthalten können. Im Jahr 1984 wurden dann Quasikristalle entdeckt, reale Festkörper, die sich aus Flüssigmetalllegierungen bilden und die Symmetrie einer Ikosaedergruppe aufweisen, die mit fünffacher Rotation einhergeht. Unterdessen entdeckte der Mathematiker Roger Penrose die Penrose-Parkettierungen der Ebene. Dabei handelt es sich um Anordnungen von Parallelogrammen, die eine Ebene mit einer fünfeckigen Langstreckenordnung abdecken. Die Legierungs-Quasikristalle sind dreidimensionale Entsprechungen zu den zweidimensionalen PenroseParkettierungen. Nach diesen Entdeckungen mussten die Mathematiker die Theorie der kristallografischen Gruppen erweitern, um auch Quasikristalle einzubeziehen. Dies ist ein umfangreiches Forschungsprojekt, das noch nicht abgeschlossen ist.

Ein vierter Scherz der Natur ist das ähnliche Verhalten von Quasikristallen und den Nullstellen der Riemannschen Zeta-Funktion. Die Nullstellen der Zeta-Funktion sind für Mathematiker insofern spannend, als sie auf einer geraden Linie liegen und niemand versteht, warum. Die Behauptung, dass sie sich alle bis auf triviale Ausnahmen auf einer geraden Linie befinden, ist die berühmte Riemannsche Vermutung. Sie zu beweisen ist seit mehr als hundert Jahren der Traum junger Mathematiker. Ich möchte nun den unerhörten Vorschlag machen, die Riemannsche Vermutung 
mithilfe von Quasikristallen zu beweisen. Diesen Vorschlag mögen die Mathematiker unter Ihnen für unseriös halten, und den Nichtmathematikern unter Ihnen erscheint er vielleicht uninteressant. Nichtsdestotrotz möchte ich ihn ernsthaft zur Diskussion stellen. Als der Physiker Leo Szilard ein junger Mann war, erschienen ihm die zehn Gebote von Moses als unzulänglich, und er verfasste eine neue Reihe von zehn Geboten, um sie zu ersetzen. Szilards zweites Gebot besagt: „Richte deine Handlungen auf ein lohnendes Ziel aus, aber frage nicht, ob sie es auch erreichen können: Sie sollen Vorbilder und Beispiele sein, nicht Mittel zum Zweck." Szilard selbst befolgte das, was er predigte. Er war der erste Physiker, der an Kernwaffen dachte, und der erste, der sich aktiv gegen deren Einsatz engagierte. Sein zweites Gebot kann hier durchaus Anwendung finden. Der Beweis der Riemannschen Vermutung ist ein lohnendes Ziel, und es ist nicht an uns zu fragen, ob wir es auch erreichen können. Ich werde Ihnen einige Anhaltspunkte nennen, um zu skizzieren, auf welche Weise es vielleicht möglich wäre. Dabei werde ich als der Mathematiker sprechen, der ich vor fünfzig Jahren war, bevor ich Physiker wurde, und zunächst auf die Riemannsche Vermutung eingehen und dann auf Quasikristalle.

Bis vor Kurzem gab es in der Welt der reinen Mathematik zwei große ungelöste Aufgaben, den Beweis des Großen Fermatschen Satzes und den Beweis der Riemannschen Vermutung. Den Großen Fermatschen Satz hat mein Kollege Andrew Wiles aus Princeton vor zwölf Jahren erledigt, und so ist nur noch die Riemannsche Vermutung übrig. Wiles' Beweis des Fermatschen Satzes war nicht nur ein technisches Kunststück. Er erforderte die Entdeckung und Erforschung eines neuen Feldes mathematischer Ideen, das weitaus umfassender und folgenreicher war als der Fermatsche Satz selbst. Es ist sehr wahrscheinlich, dass jeglicher Beweis der Riemannschen Vermutung ebenfalls zu einem tieferen Verständnis vieler verschiedener Bereiche der Mathematik und vielleicht auch der Physik führen wird. Die Riemannsche Zeta-Funktion und andere ähnliche Zeta-Funktionen kommen überall in der Zahlentheorie, in der Theorie dynamischer Systeme, in der Geometrie, in der Funktionstheorie und in der Physik vor. Die Zeta-Funktion steht an einem Kreuzungspunkt, von dem aus Wege in viele Richtungen führen. Ein Beweis der Vermutung wird alle diese Verbindungen beleuchten. Wie jeder ernsthafte Student der reinen Mathematik träumte ich in jungen Jahren davon, die Riemannsche Vermutung zu beweisen. Ich hatte einige vage Ideen, die meiner Ansicht nach zu einem Beweis führen könnten. Nach der Entdeckung von Quasikristallen sind meine Ideen in den letzten Jahren etwas weniger vage geworden. Ich möchte sie hier vorstellen und jedem jungen Mathematiker mit Ambitionen auf eine Fields-Medaille ans Herz legen, über sie nachzudenken.

Quasikristalle können in Räumen mit einer, zwei oder drei Dimensionen existieren. Vom Standpunkt der Physik aus sind die dreidimensionalen Quasikristalle am interessantesten, weil sie in unserer dreidimensionalen Welt angesiedelt sind und experimentell erforscht werden können. Vom Standpunkt eines Mathematikers aus sind eindimensionale Quasikristalle viel interessanter als zwei- oder dreidimensionale, weil sie in weitaus größerer Vielfalt existieren. Die mathematische Definition eines Quasikristalls lautet wie folgt: Ein Quasikristall ist eine Anordnung von diskreten Punktmassen, deren Fourier-Transformation eine Anordnung von diskreten Punktfrequenzen ist. Oder, kurz gesagt, ein Quasikristall ist eine reine Punktanordnung, die ein reines Punktspektrum besitzt. Diese Definition schließt als Sonderfall die normalen Kristalle ein, die periodische Anordnungen mit periodischen Spektren sind.

Außer den normalen Kristallen gibt es nur wenige verschiedene dreidimensionale Quasikristalle, die alle mit der Ikosaedergruppe verbunden sind. Die zweidimensionalen Quasikristalle sind vielfältiger, wobei mit jedem regelmäßigen Vieleck in einer Ebene ungefähr ein bestimmter Typ verbunden ist. Der zweidimensionale Quasikristall mit Fünfecksymmetrie ist die berühmte Penrose-Parkettierung der Ebene. Die eindimensionalen Quasikristalle schließlich haben eine weitaus reichere Struktur, weil sie an keinerlei Rotationssymmetrien gebunden sind. Soweit ich weiß, existiert keine vollständige Aufzählung eindimensionaler Quasikristalle. Es ist bekannt, dass es zu jeder Pisot-VijayaraghavanZahl oder PV-Zahl einen einzigartigen Quasikristall gibt. Eine PV-Zahl ist eine reelle algebraische ganze Zahl, eine Wurzel einer Polynomgleichung mit ganzzahligen Koeffizienten, wobei alle anderen Wurzeln einen absoluten Wert von weniger als eins haben [1]. Die Menge aller PV-Zahlen ist unendlich und hat eine bemerkenswerte topologische Struktur. Die Menge aller eindimensionalen Quasikristalle hat eine Struktur, die mindestens so reichhaltig ist wie die Menge aller PV-Zahlen und vermutlich noch viel reichhaltiger. Es ist nicht ganz sicher, aber wahrscheinlich, dass ein gigantisches Universum eindimensionaler Quasikristalle, die nicht mit PV-Zahlen verbunden sind, darauf wartet, entdeckt zu werden. Und nun zur Verbindung der eindimensionalen Quasikristalle mit der Riemannschen Vermutung. Wenn die Riemannsche Vermutung zutrifft, dann bilden die Nullstellen der Zeta-Funktion einen eindimensionalen Quasikristall gemäß der Definition. Sie stellen eine Anordnung von Punktmassen auf einer geraden Linie dar, und ihre Fourier-Transformation ist ebenfalls eine Anordnung von Punktmassen, einer bei jedem Logarithmus von gewöhnlichen Primzahlen und Primzahlpotenzen. Mein Freund Andrew Odlyzko hat eine wunderbare Computerberechnung für die Fourier-Transformation der Nullstellen der ZetaFunktion veröffentlicht [6]. Die Berechnung zeigt genau die erwartete Struktur der Fourier-Transformation, die bei jedem Logarithmus einer Primzahl oder Primzahlpotenz und nirgendwo sonst eine sehr deutliche Diskontinuität zeigt.

Ich möchte nun Folgendes vorschlagen. Nehmen wir einmal an, wir wüssten nicht, dass die Riemannsche Vermutung wahr ist. Nähern wir uns dem Problem von der anderen Seite und versuchen uns eine vollständige Aufzählung und Klassifizierung der eindimensionalen Quasikristalle zu verschaffen. Das heißt, wir zählen und klassifizieren alle Punktanordnungen, die ein diskretes Punktspektrum haben. Ein solches Sammeln und Klassifizieren neuer Arten von Objekten ist eine typisch baconianische Tätigkeit, die besonders für mathematische Frösche geeignet ist. Wir werden dabei die bekannten, mit den PV-Zahlen verbundenen Qua- 
sikristalle finden und darüber hinaus ein ganzes Universum anderer bekannter und unbekannter Quasikristalle. Unter diesen zahlreichen anderen Quasikristallen suchen wir einen, der der Riemannschen Zeta-Funktion entspricht, und einen für jede andere Zeta-Funktion, die der Riemannschen Zeta-Funktion ähnelt. Angenommen, wir finden in unserer Auflistung einen Quasikristall mit Eigenschaften, die ihn mit den Nullstellen der Riemannschen Zeta-Funktion identifizieren. Dann haben wir die Riemannsche Vermutung bewiesen und können auf den Telefonanruf warten, der uns die Verleihung der Fields-Medaille ankündigt.

Das sind natürlich nur Wunschträume. Eindimensionale Quasikristalle zu klassifizieren ist ungeheuer schwierig, wahrscheinlich mindestens ebenso schwierig wie die Aufgabe, für deren Bewältigung Andrew Wiles sieben Jahre brauchte. Aber aus baconianischer Perspektive ist die ganze Geschichte der Mathematik eine Geschichte ungeheuer schwieriger Aufgaben, die von jungen Menschen gelöst wurden, die zu wenig wussten, um zu verstehen, dass sie unlösbar waren. Die Klassifizierung von Quasikristallen ist ein lohnendes Ziel, das sich womöglich sogar als erreichbar erweist. Dermaßen schwierige Probleme werden jedoch nicht von alten Männern wie mir gelöst. Ich überlasse diese Aufgabe den jungen Fröschen im Publikum.

\section{Abram Besicovitch und Hermann Weyl}

Lassen Sie mich Ihnen nun einige bedeutende Frösche und Vögel vorstellen, die ich persönlich kannte. Ich kam 1941 als Student an die Universität Cambridge und hatte das große Glück, dass der russische Mathematiker Abram Samoilowitsch Besikowitsch mein Betreuer war. Weil wir uns mitten im Zweiten Weltkrieg befanden, gab es in Cambridge insgesamt nur sehr wenige Studenten und fast keine graduierten. Obwohl ich erst siebzehn Jahre alt war und Besikowitsch bereits ein berühmter Professor, schenkte er mir sehr viel Zeit und Aufmerksamkeit, und wir wurden lebenslang Freunde. Besikowitsch prägte die Art und Weise, in der ich zu arbeiten und über Mathematik nachzudenken begann. Er hielt wunderbare Vorträge über Maßtheorie und Integration und lächelte liebenswürdig, wenn wir uns über seinen herrlich falschen Gebrauch der englischen Sprache lustig machten. Ich erinnere mich nur an eine einzige Situation, in der er sich über unser Lachen ärgerte. Er schwieg eine Zeit lang und sagte dann: „Gentlemen. Fünfzig Millionen Engländer sprechen das Englisch, das Sie sprechen. Hundertfünfzig Millionen Russen sprechen das Englisch, das ich spreche."

Besikowitsch war ein Frosch und wurde als junger Mann dadurch berühmt, dass er das als Kakeya-Problem bekannte Problem der einfachen Flächengeometrie löste. Das KakeyaProblem bestand in Folgendem: Eine Strecke der Länge eins darf sich in einer Ebene frei bewegen, während sie sich um 360 Grad dreht. Wie groß ist die kleinstmögliche Fläche der Ebene, die sie während ihrer Drehung überstreichen kann? Diese Frage wurde im Jahr 1917 von dem japanischen Mathematiker Kakeya gestellt und blieb zehn Jahre lang ein berühmtes ungelöstes Problem. George Birkhoff, der zu dieser Zeit führende amerikanische Mathematiker, verkündete öffentlich, das Kakeya-Problem und das Vier-Farben-Problem seien die bedeutendsten noch nicht gelösten Probleme der Zeit. Allgemein wurde angenommen, die Mindestfläche betrage $\pi / 8$, was der Fläche einer dreispitzigen Hypozykloide entspricht. Bei der dreispitzigen Hypozykloide handelt es sich um eine wunderbare dreispitzige Kurve. Sie ist die Kurve, die von einem Punkt auf dem Umfang eines Kreises mit einem Radius von einem Viertel beschrieben wird, wenn der Kreis über die Innenseite eines festen Kreises mit einem Radius von drei Vierteln rollt. Die Strecke der Länge eins kann sich drehen und bleibt dabei stets tangential zur Hypozykloide, wobei ihre beiden Endpunkte ebenfalls auf der Hypozykloide liegen. Dieses Bild der sich drehenden Strecke, die die Innenseite der Hypozykloide an drei Punkten berührt, war so elegant, dass die meisten Leute glaubten, sie müsse die minimale Fläche angeben. Dann überraschte Besikowitsch alle, indem er bewies, dass die Fläche, die von der Strecke bei ihrer Drehung überstrichen wird, für jedes positive $\varepsilon$ kleiner als $\varepsilon$ sein kann. Besikowitsch hatte das Problem tatsächlich bereits 1920 gelöst, bevor es berühmt wurde und sogar ohne zu wissen, dass Kakeya es vorgestellt hatte. Im Jahr 1920 veröffentlichte er die Lösung in russischer Sprache in der Zeitschrift der Permer Gesellschaft

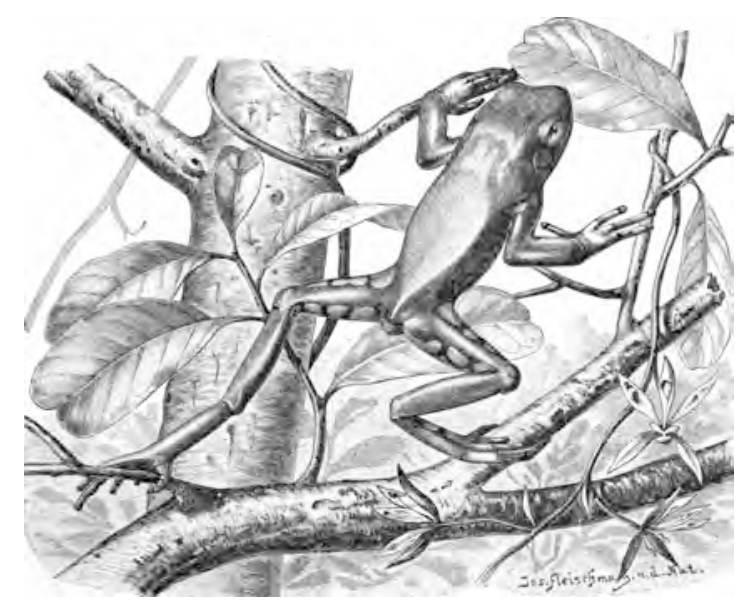




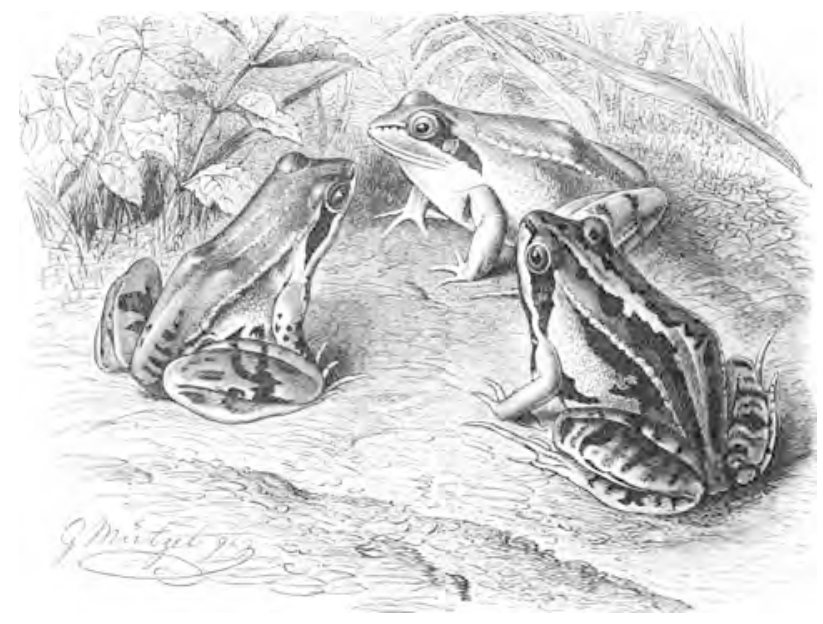

für Physik und Mathematik, einer Zeitschrift, die anderswo kaum gelesen wurde. Die Universität in Perm, einer 1100 Kilometer östlich von Moskau gelegenen Stadt, war nach der russischen Revolution kurzzeitig ein Zufluchtsort für viele herausragende Mathematiker. Sie brachten zwei Bände der Zeitschrift heraus, bevor sie in den Wirren von Revolution und Bürgerkrieg unterging. Außerhalb Russlands war die Zeitschrift nicht nur unbekannt, sondern auch nicht zugänglich. Besikowitsch verließ Russland 1925 und kam nach Kopenhagen, wo er von dem berühmten Kakeya-Problem hörte, das er fünf Jahre zuvor gelöst hatte. Erneut veröffentlichte er die Lösung, diesmal auf Englisch in der $\mathrm{Ma}$ thematischen Zeitschrift. Das Kakeya-Problem, wie Kakeya es formuliert hatte, war ein typisches Froschproblem, ein konkretes Problem ohne große Verbindung zum Rest der Mathematik. Besikowitsch lieferte dafür eine elegante und profunde Lösung, die eine Verbindung zu allgemeinen Sätzen über die Struktur von Punktmengen in einer Ebene zum Vorschein brachte.

Der Stil Besikowitschs lässt sich am besten anhand seiner drei klassischen Abhandlungen mit dem Titel „On the fundamental geometric properties of linearly measurable plane sets of points“ („Über die grundlegenden geometrischen Eigenschaften linear messbarer ebener Punktmengen") nachvollziehen, die in den Jahren 1928, 1938 und 1939 in den Mathematischen Annalen veröffentlicht wurden. In diesen Beiträgen bewies er, dass jede linear messbare Punktmenge in der Ebene in eine reguläre und eine irreguläre Komponente teilbar ist, dass die reguläre Komponente fast überall eine Tangente hat und die Projektion der irregulären Komponente in fast alle Richtung Maß Null besitzt. Grob gesagt sieht die reguläre Komponente wie eine Ansammlung von Ansammlung stetiger Kurven aus, während die irreguläre Komponente überhaupt nicht wie eine überhaupt nicht wie eine stetige Kurve aussieht. aussieht. Die Existenz und die Eigenschaften der irregulären Komponente stehen im Zusammenhang mit Besikowitschs Lösung des KakeyaProblems. Eine der Aufgaben, die er mir zur Bearbeitung gab, war die Zerlegung messbarer Mengen in reguläre und irreguläre Komponenten in Räumen höherer Dimension. Ich machte bei der Lösung dieser Aufgabe keinerlei Fortschrit- te, wurde aber dauerhaft von Besikowitschs Stil geprägt. Dieser Stil ist ein architektonischer. Besikowitsch konstruiert aus einfachen Elementen ein kompliziertes filigranes, meist hierarchisch strukturiertes Gebäude, und wenn dieses dann fertig ist, führt es mit einfachen Argumenten zu einem unerwarteten Ergebnis. Jeder Besikowitsch-Beweis ist ein Kunstwerk, das ebenso sorgfältig konstruiert ist wie eine Fuge von Bach.

Ein paar Jahre nach meiner Ausbildung bei Besikowitsch kam ich nach Princeton und lernte Hermann Weyl kennen. Weyl war ebenso ein typischer Vogel, wie Besikowitsch ein typischer Frosch war. Ich hatte das Glück, ein Jahr lang gemeinsam mit Weyl am Princeton Institute for Advanced Study zu arbeiten, bevor er sich aus dem Institut zurückzog und in seine alte Heimat nach Zürich zurückkehrte. Er mochte mich, weil ich in diesem Jahr in den Annals of Mathematics Beiträge über die Zahlentheorie und in der Physical Review Beiträge über die Quantentheorie der Strahlung veröffentlichte. Er war einer der wenigen lebenden Menschen, die in beiden Fachgebieten zu Hause waren, und begrüßte mich am Institut in der Hoffnung, dass ich wie er selbst ein Vogel wäre. Doch er wurde enttäuscht. Ich war und blieb ein Frosch. Obwohl ich in den verschiedensten Schlammlöchern herumstocherte, hatte ich immer nur jeweils eines davon im Blick und hielt nicht nach Verbindungen zwischen ihnen Ausschau. Für mich waren Zahlentheorie und Quantentheorie unterschiedliche Welten mit unterschiedlichen Reizen. Ich betrachtete sie nicht wie Weyl in der Hoffnung, Anhaltspunkte für einen großen Entwurf zu finden.

Weyls bedeutender Beitrag zur Quantentheorie der Strahlung war seine Erfindung der Eichfelder. Die Idee der Eichfelder hatte eine seltsame Geschichte. Weyl erfand sie 1918 in seiner vereinheitlichten Theorie der allgemeinen Relativitätstheorie und des Elektromagnetismus als klassische Felder [7]. Er nannte sie „Eichfelder“, weil sie mit der Nichtintegrierbarkeit von Längenmessungen zu tun hatten. Seine vereinheitlichte Theorie wurde von Einstein umgehend öffentlich zurückgewiesen. Nach diesem Donnerschlag von oben gab Weyl seine Theorie nicht auf, wandte sich aber anderen Dingen zu. Die Theorie hatte keine überprüfbaren experimentellen Konsequenzen. Im Jahr 1929 dann, nach- 
dem von anderen die Quantenmechanik erfunden worden war, erkannte Weyl, dass seine Eichfelder wesentlich besser in die Welt der Quanten passten als in die klassische Welt [8]. Um ein klassisches Eichmaß in ein Quanten-Eichmaß zu verwandeln, musste er lediglich aus reellen Zahlen komplexe Zahlen machen. In der Quantenmechanik besitzt jedes Quant elektrischer Ladung eine komplexe Wellenfunktion mit einer Phase, und das Eichfeld ist mit der Nichtintegrierbarkeit der Messung der Phasen behaftet. Das Eichfeld konnte nun mit dem elektromagnetischen Potenzial exakt identifiziert werden, und der Ladungserhaltungssatz erschien als Folgerung aus der lokalen Phaseninvarianz der Theorie.

Weyl starb vier Jahre, nachdem er von Princeton nach Zürich zurückgekehrt war, und ich schrieb für die Zeitschrift Nature einen Nachruf auf ihn [3]. „Unter allen Mathematikern, deren Berufsleben im zwanzigsten Jahrhundert begann“, schrieb ich, „leistete Hermann Weyl in den meisten unterschiedlichen Bereichen wichtige Beiträge. Nur er konnte den Vergleich mit den letzten großen Universalmathematikern des neunzehnten Jahrhunderts, Hilbert und Poincaré, bestehen. Solange er lebte, verkörperte er den lebendigen Kontakt zwischen den wichtigsten Entwicklungen in der reinen Mathematik und in der theoretischen Physik. Nun, da er tot ist, ist dieser Kontakt abgebrochen, und unsere Hoffnungen, das physikalische Universum durch die direkte Anwendung der kreativen mathematischen Fantasie zu begreifen, haben sich einstweilen zerschlagen." Ich trauerte um ihn, aber ich hatte nicht das Verlangen, seinen Traum weiterzuverfolgen. Ich war froh darüber, dass die reine Mathematik und die Physik in entgegengesetzten Richtungen voranschritten.

Der Nachruf endete mit einer skizzenhaften Beschreibung des Menschen Weyl: „Charakteristisch für Weyl war ein Sinn für Ästhetik, der sein Denken bei allen Themen beherrschte. Er sagte einmal halb im Scherz zu mir: ,Meine Arbeit versuchte stets, das Wahre mit dem Schönen zu verbinden; aber wenn ich mich für eines von beiden entscheiden musste, wählte ich normalerweise das Schöne. Diese Bemerkung bringt seine Persönlichkeit perfekt auf den Punkt. Sie zeigt seinen tiefen Glauben an eine grundlegende Harmonie der Natur, in der sich Gesetze notwendigerweise in einer mathematisch schönen Form ausdrücken lassen. Sie zeigt auch seine Einsicht in die Schwäche des Menschen und seinen Humor, der ihn von jeglicher Wichtigtuerei abhielt. Seine Freunde in Princeton werden ihn so in Erinnerung behalten, wie ich ihn im April letzten Jahres beim Frühlingsball des Institute for Advanced Study zum letzten Mal sah: als großen, heiteren Mann, der sich prächtig amüsiert und dessen Aufgeräumtheit und Leichtfüßigkeit seine neunundsechzig Jahre nicht vermuten lassen." Die fünfzig Jahre nach Weyls Tod waren ein goldenes Zeitalter für die Experimentalphysik und die beobachtende Astronomie, ein goldenes Zeitalter für baconianische, Fakten sammelnde Reisende, für Frösche, die kleine Fleckchen des Sumpfes, in dem wir leben, erforschen. Im Verlauf dieser fünfzig Jahre sammelten die Frösche detailliertes Wissen über die verschiedensten kosmischen Strukturen und die verschiedensten Teilchen und Wechselwirkungen. Mit der fortschreitenden Erforschung neuer Gebiete wurde das Universum immer komplizierter. Statt eines großen Entwurfs, der die Einfachheit und Schönheit der Weyl'schen Mathematik aufweisen würde, fanden die Forscher seltsame Objekte wie Quarks und Gammablitze, merkwürdige Konzepte wie die Supersymmetrie und multiple Universen. Gleichzeitig wurde auch die Mathematik durch die Erforschung von Chaosphänomenen und vielen anderen neuen, durch den Einsatz von elektronischen Computern erschlossenen Gebieten immer komplizierter. Die Mathematiker entdeckten das zentrale ungelöste Problem der Berechenbarkeit, die durch die Aussage „P ist nicht gleich NP“ dargestellte Vermutung. Sie besagt, dass es mathematische Probleme gibt, die im Einzelfall schnell gelöst werden können, aber nicht durch einen schnellen, auf alle Fälle anwendbaren Algorithmus. Das bekannteste Beispiel für ein solches Problem ist das Problem des Handlungsreisenden, bei dem es darum geht, für einen Handlungsreisenden die kürzeste Route durch eine Reihe von Städten zu finden, wobei die Entfernung zwischen jedem Städtepaar bekannt ist. Alle Experten sind der Meinung, dass die Vermutung zutrifft und das Problem des Handlungsreisenden ein Beispiel für ein Problem ist, das P, aber nicht NP ist. Doch niemand hat auch nur den Schimmer einer Idee, wie sich das beweisen ließe. Dies ist ein ungelöstes Problem, das in Hermann Weyls mathematischem Universum des neunzehnten Jahrhunderts nicht einmal hätte formuliert werden können.

\section{Frank Yang und Yuri Manin}

Die letzten fünfzig Jahre waren für Vögel eine schwierige Zeit. Aber auch in schwierigen Zeiten gibt es für sie genug zu tun, und es fanden sich Vögel, die den Mut hatten, es anzugehen. Kurz nachdem Weyl Princeton verlassen hatte, kam Frank Yang aus Chicago und zog in Weyls altes Haus ein. Yang trat als der führende Vogel meiner Generation von Physikern an Weyls Stelle. Noch zu Weyls Lebzeiten entwickelten Yang und sein Schüler Robert Mills die Yang-Mills-Theorie der nichtabelschen Eichfelder, eine unglaublich elegante Erweiterung der Weyl'schen Eichfeldidee [11]. Weyls Eichfeld war eine klassische Größe, die dem Kommutativgesetz der Multiplikation genügte. Die Yang-Mills-Theorie beinhaltete drei Eichfelder, die nicht kommutierten. Sie erfüllten die Kommutationsregeln der drei Komponenten eines quantenmechanischen Spins, die Erzeugende der einfachsten nichtabelschen Lie-Algebra $A_{2}$ sind. Diese Theorie wurde später verallgemeinert, sodass die Eichfelder Erzeuger einer beliebigen endlichdimensionalen Lie-Algebra sein konnten. Mit dieser Verallgemeinerung lieferte die Yang-Mills-Eichfeldtheorie den Rahmen für das Modell aller bekannten Teilchen und Wechselwirkungen, das heute als Standardmodell der Teilchenphysik bekannt ist. Yang gab ihm den letzten Schliff, indem er zeigte, dass auch Einsteins Gravitationstheorie in diesen Rahmen passt, wobei das Christoffel'sche Dreiindexsymbol die Rolle des Eichfeldes übernimmt [10].

In einem Zusatz zu seinem Aufsatz von 1918, der 1955 in den anlässlich seines siebzigsten Geburtstags veröffent- 


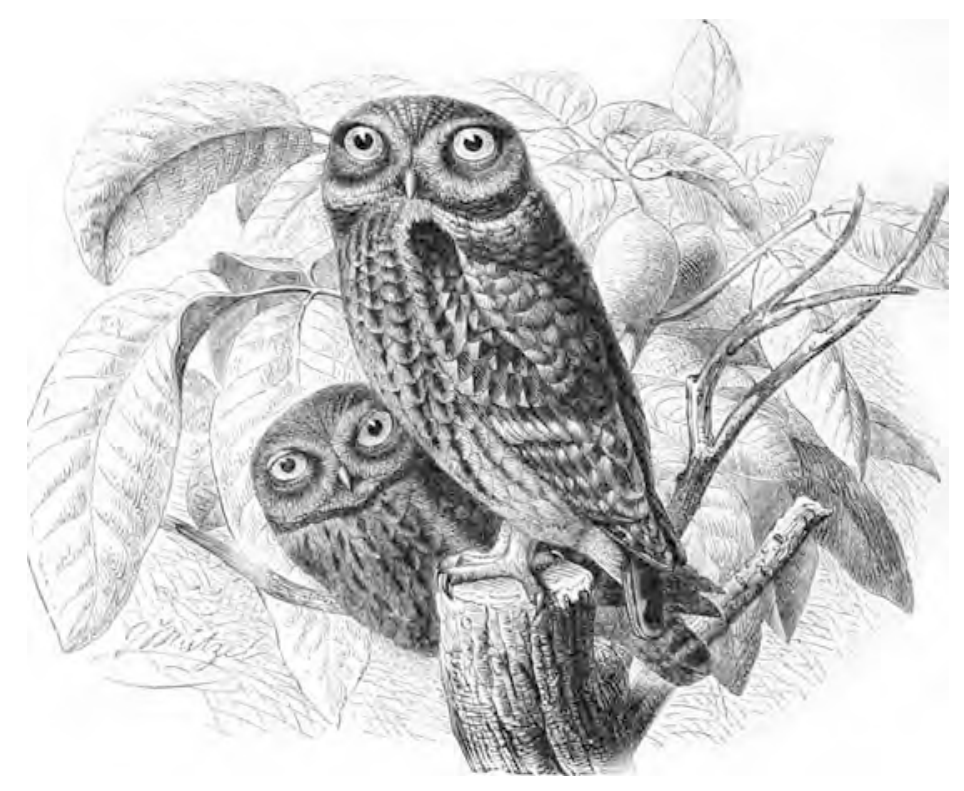

lichten Band ausgewählter Schriften aufgenommen wurde, äußerte Weyl seine abschließenden Gedanken zu den Eichfeldtheorien [12]: „Das stärkste Argument für meine Theorie schien dies zu sein, daß die Eichinvarianz dem Prinzip von der Erhaltung der elektrischen Ladung so entspricht wie die Koordinaten-Invarianz dem Erhaltungssatz von Energie-Impuls." Dreißig Jahre später war Yang bei den Feierlichkeiten zu Weyls hundertstem Geburtstag in Zürich. In seiner Rede [12] zitierte er diese Anmerkung als Beweis dafür, dass Weyl an der Idee der Eichinvarianz als verbindendem Prinzip der Physik festhielt. Yang führte weiter aus: „Symmetrie, Lie-Gruppen und Eichinvarianz spielen heute aufgrund theoretischer und experimenteller Entwicklungen anerkanntermaßen eine wesentliche Rolle bei der Bestimmung der Grundkräfte des physikalischen Universums. Ich habe dies das Prinzip, dass die Symmetrie die Wechselwirkung bestimmt, genannt." Diese Vorstellung, dass die Symmetrie die Wechselwirkung bestimmt, ist Yangs Verallgemeinerung von Weyls Anmerkung. Weyl hatte festgestellt, dass die Eichinvarianz eng mit den physikalischen Erhaltungssätzen verbunden ist. Darüber konnte er nicht hinausgehen, da er nur die Eichinvarianz der kommutierenden abelschen Felder kannte. Yang stärkte diese Verbindung erheblich, indem er nichtabelsche Eichfelder einführte. Mit nichtabelschen Eichfeldern, die nichttriviale Lie-Algebren hervorbringen, werden die möglichen Formen der Wechselwirkung zwischen den Feldern einzigartig, sodass die Symmetrie die Wechselwirkung bestimmt. Dieser Gedanke ist Yangs bedeutendster Beitrag zur Physik. Es ist der Beitrag eines Vogels, der hoch über dem Regenwald der kleinen Probleme schwebt, in dem die meisten von uns ihr Leben verbringen.

Ein weiterer Vogel, vor dem ich großen Respekt habe, ist der russische Mathematiker Yuri Manin, der vor Kurzem einen wunderbaren Aufsatzband mit dem Titel Mathematics as Metaphor („Mathematik als Metapher“) [5] veröffentlicht hat. Das Buch wurde in Moskau auf Russisch und von der American Mathematical Society auf Englisch herausgebracht. Für die englische Version habe ich ein Vorwort geschrieben, aus dem ich hier kurz zitieren möchte. „Mathematik als Metapher ist ein guter Slogan für Vögel. Er besagt, dass die tiefgründigsten mathematischen Gedanken diejenigen sind, die eine Gedankenwelt mit einer anderen verbinden. Im 17. Jahrhundert hat Descartes die ungleichen Welten Algebra und Geometrie durch sein Konzept der Koordinaten verbunden, und Newton verband die Welten der Geometrie und der Dynamik durch das Konzept der Fluxionen, das heute Differenzialrechnung genannt wird. Im 19. Jahrhundert verknüpfte Boole mit seinem Konzept der symbolischen Logik die Welten der Logik und der Algebra, und Riemann verband die Welten der Geometrie und der Analysis durch sein Konzept der Riemannschen Flächen. Koordinaten, Fluxionen, symbolische Logik und Riemannsche Flächen sind sämtlich Metaphern, die die Bedeutung von Wörtern von bekannten auf unbekannte Zusammenhänge ausdehnen. Manin sieht die Zukunft der Mathematik in der Erforschung von Metaphern, die bereits sichtbar, aber noch nicht verstanden sind. Die tiefgreifendste derartige Metapher ist die strukturelle Ähnlichkeit zwischen Zahlentheorie und Physik. Auf beiden Gebieten gibt es für ihn verheißungsvolle Anzeichen für parallele Konzepte, Symmetrien, die das Stetige mit dem Unstetigen verbinden. Er erwartet eine Vereinheitlichung, die er die Quantisierung der Mathematik nennt.

Manin ist mit der baconianischen Darstellung, dass Hilbert die Richtung für die Mathematik des zwanzigsten Jahrhunderts vorgab, als er 1900 auf dem Internationalen Mathematikerkongress in Paris seine berühmte Liste der dreiundzwanzig ungelösten Probleme präsentierte, nicht einverstanden. Für Manin lenkten Hilberts Probleme von den zentralen Themen der Mathematik ab. Die wichtigen Fortschritte in der Mathematik resultieren seiner Ansicht nach aus Programmen, nicht aus Problemen. Probleme werden normalerweise gelöst, indem alte Ideen auf neue Art ange- 
wandt werden. Forschungsprogramme sind die Geburtsstätten neuer Ideen. Manin betrachtet das Bourbaki-Programm, das die gesamte Mathematik in abstrakterer Sprache neu formuliert hat, als Quelle vieler neuer Ideen des zwanzigsten Jahrhunderts. Das Langlands-Programm, das die Zahlentheorie mit der Geometrie zusammenführt, sieht er als eine vielversprechende Quelle für neue Ideen im einundzwanzigsten Jahrhundert. Menschen, die berühmte ungelöste Probleme lösen, erhalten vielleicht wichtige Preise, aber die wahren Pioniere sind Menschen, die neue Programme ins Leben rufen."

Die russische Version von Mathematics as Metaphor enthält zehn Kapitel, die in der englischen Version weggelassen wurden. Die American Mathematical Society befand, dass diese Kapitel für englischsprachige Leser nicht von Interesse seien. Diese Streichungen sind in zweifacher Hinsicht bedauerlich. Erstens erhalten die Leser der englischen Version nur ein ausschnitthaftes Bild von Manin, der mit seinen vielfältigen, weit über die Mathematik hinausgehenden Interessen unter Mathematikern vermutlich einzigartig ist. Zweitens bekommen sie auf diese Weise nur einen eingeschränkten Einblick in die russische Kultur, die weniger segmentiert ist als die englischsprachige Kultur und in der Mathematiker einen engeren Kontakt zu Historikern, Künstlern und Dichtern haben.

\section{John von Neumann}

Eine weitere bedeutende Persönlichkeit in der Mathematik des zwanzigsten Jahrhunderts war John von Neumann. Von Neumann war ein Frosch, der seine herausragenden technischen Fertigkeiten einsetzte, um Probleme aus vielen Bereichen der Mathematik und der Physik zu lösen. Er fing mit den Grundlagen der Mathematik an und fand das erste zufriedenstellende axiomatische System für die Mengenlehre, wobei er die logischen Paradoxien umging, auf die Cantor bei seinen Versuchen, mit unendlichen Mengen und unendlichen Zahlen umzugehen, gestoßen war. Von Neumanns Axiome wurden einige Jahre später von seinem Freund Kurt Gödel, einem Vogel, für den Beweis der Existenz unentscheidbarer Sätze in der Mathematik verwendet. Gödels Sätze eröffneten den Vögeln einen neuen Blick auf die Mathematik. Ihm zufolge war die Mathematik kein einheitliches, mit einem einzigen Wahrheitsbegriff verbundenes Gebäude mehr, sondern ein Archipel einzelner Bauten mit verschiedenen axiomatischen Systemen und unterschiedlichen Wahrheitsbegriffen. Gödel zeigte, dass die Mathematik unerschöpflich ist. Egal, welches axiomatische System man zugrunde legt, Vögel finden immer Fragen, die diese Axiome nicht beantworten können.

Von Neumann ging von den Grundlagen der Mathematik weiter zu den Grundlagen der Quantenmechanik. Um der Quantenmechanik ein solides mathematisches Fundament zu verschaffen, entwarf er die großartige Theorie der Operatorenringe. Jede beobachtbare Größe wird durch einen linearen Operator dargestellt, und die Besonderheiten des Quantenverhaltens werden durch die Operatoralgebra genau abgebildet. Wie Newton die Analysis erfand, um die klassische Dynamik zu beschreiben, erfand von Neumann die Operatorenringe, um die Quantendynamik zu beschreiben.

Von Neumann leistete auf verschiedenen anderen Gebieten grundlegende Beiträge, insbesondere zur Spieltheorie und zur Konstruktion von Digitalrechnern. Die letzten zehn Jahre seines Lebens befasste er sich intensiv mit Computern. Sein Interesse daran war so stark, dass er beschloss, nicht nur die Konstruktion von Computern zu erforschen, sondern selber einen Computer mit Hard- und Software zu bauen und für die wissenschaftliche Arbeit zu nutzen. Ich erinnere mich lebhaft an die Anfänge seines Computerprojekts am Institute for Advanced Study in Princeton. $\mathrm{Zu}$ dieser Zeit hatte von Neumann zwei zentrale wissenschaftliche Interessen: Wasserstoffbomben und Meteorologie. Nachts benutzte er seinen Computer für Berechnungen im Zusammenhang mit Wasserstoffbomben und tagsüber für die Meteorologie. Die meisten Leute, die sich tagsüber im Computergebäude aufhielten, waren Meteorologen. Ihr Direktor war Jule Charney. Charney war ein echter Meteorologe, gebührend bescheiden im Umgang mit den unergründlichen Geheimnissen des Wetters und skeptisch gegenüber der Fähigkeit des Computers, diese Geheimnisse zu lüften. John von Neumann war weniger bescheiden und weniger skeptisch. Ich hörte einen Vortrag von ihm über die Ziele seines Projekts. Wie immer sprach er dabei mit großer Zuversicht. Er sagte: „Der Computer wird uns in die Lage versetzen, die Atmosphäre jederzeit in stabile und instabile Bereiche zu unterteilen. Stabile Bereiche können wir vorhersagen. Instabile Bereiche können wir beherrschen." Von Neumann glaubte, dass jeder instabile Bereich durch eine geschickt eingesetzte kleine Störung so verschoben werden könnte, dass er sich in jede gewünschte Richtung bewegte. Diese kleine Störung sollte durch eine Flotte von Flugzeugen erzeugt werden, die Rauchgeneratoren an Bord hatten, um an den für eine wirksame Störung geeigneten Stellen Sonnenstrahlen zu absorbieren und die Temperatur zu erhöhen oder zu senken. Vor allem sollte man einen aufziehenden Hurrikan aufhalten können, indem man die Position einer Instabilität rechtzeitig identifizierte und diese Luftschicht dann abkühlte, bevor sie aufstieg und einen Strudel ausbildete. 1950 sagte von Neumann, es werde nur zehn Jahre dauern, Computer zu bauen, die leistungsfähig genug seien, um die stabilen und instabilen Bereiche der Atmosphäre genau zu bestimmen. Hätte man dann erst einmal die genaue Diagnose, werde man innerhalb kürzester Zeit auch die Kontrolle haben. Er erwartete, dass die praktische Kontrolle des Wetters in den 196oer-Jahren eine Routinetätigkeit sein würde.

Von Neumann lag natürlich falsch. Und zwar deshalb, weil er noch nichts vom Chaos wusste. Heute ist bekannt, dass die Bewegung der Atmosphäre bei lokaler Instabilität sehr oft chaotisch ist. „Chaotisch“ bedeutet, dass Bewegungen, die dicht nebeneinander beginnen, mit der Zeit exponentiell auseinanderlaufen. Wenn die Bewegung chaotisch ist, ist sie unvorhersehbar, und eine kleine Störung versetzt sie nicht in eine stabile Bewegung, die sich vorhersagen lässt. Eine kleine Störung bringt sie normalerweise in eine andere chaotische Bewegung, die ebenso unvorhersehbar ist. Deshalb funktioniert von Neumanns Strategie, das Wetter zu 


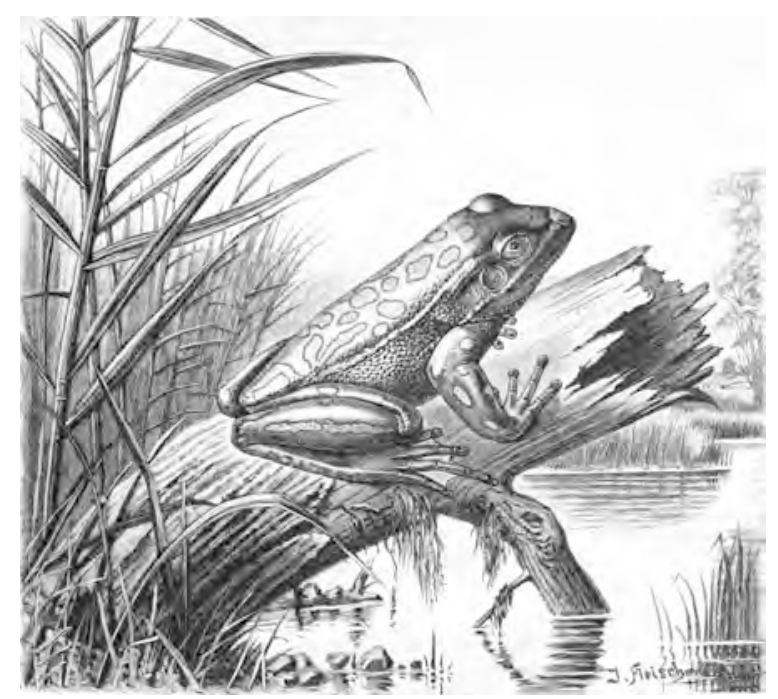

kontrollieren, nicht. Er war eben ein großer Mathematiker, wenn auch nur ein mittelmäßiger Meteorologe.

Edward Lorenz entdeckte 1963, dass die Lösungen von meteorologischen Gleichungen oft chaotisch sind. Das war sechs Jahre nach von Neumanns Tod. Lorenz war Meteorologe und wird allgemein als Entdecker des Chaos angesehen. Er erkannte die Chaosphänomene im meteorologischen Bereich und gab ihnen ihre modernen Namen. Tatsächlich aber hatte ich bereits 1943, zwanzig Jahre bevor Lorenz sie entdeckte, eine Beschreibung derselben Phänomene in einem Vortrag von Mary Cartwright in Cambridge gehört, einer Mathematikerin, die 1998 im Alter von 97 Jahren starb. Sie gab diesen Phänomenen andere Namen, aber es waren dieselben Phänomene. Sie entdeckte sie in den Lösungen der Van-der-Pol-Gleichung, die die Schwingungen eines nichtlinearen Verstärkers beschreiben [2]. Die Van-der-PolGleichung war im Zweiten Weltkrieg von Bedeutung, weil nichtlineare Verstärker die Transmitter in frühen Radarsystemen mit Strom versorgten. Die Transmitter verhielten sich unberechenbar, und die Air Force beschuldigte die Hersteller, defekte Verstärker herzustellen. Mary Cartwright wurde gebeten, das Problem zu analysieren. Sie konnte zeigen, dass die Hersteller keine Schuld traf. Sie wies nach, dass die Ursache in der Van-der-Pol-Gleichung lag. Die Lösungen der Van-der-Pol-Gleichung zeigen genau das chaotische Verhalten, das die Luftwaffe beanstandete. Sieben Jahre bevor ich von Neumann über die Kontrolle des Wetters sprechen hörte, hörte ich von Mary Cartwright alles über das Chaos, aber ich war nicht weitsichtig genug, um die Verbindung herzustellen. Es kam mir nie in den Sinn, dass das unberechenbare Verhalten der Van-der-Pol-Gleichung etwas mit Meteorologie zu tun haben könnte. Wäre ich ein Vogel und kein Frosch gewesen, hätte ich wahrscheinlich einen Zusammenhang gesehen und von Neumann viele Schwierigkeiten erspart. Hätte er $195^{\circ}$ vom Chaos gewusst, hätte er wahrscheinlich gründlich darüber nachgedacht und 1954 etwas Entscheidendes dazu sagen können.

Von Neumann geriet am Ende seines Lebens in Schwierigkeiten, weil jeder von ihm erwartete, wie ein Vogel zu fliegen, er aber in Wirklichkeit ein Frosch war. Im Jahr 1954 gab es in Amsterdam einen Internationalen Mathematikerkongress. Diese Kongresse finden nur alle vier Jahre statt, und es ist eine große Ehre, bei der Eröffnungssitzung sprechen zu dürfen. Die Organisatoren des Amsterdamer Kongresses luden von Neumann ein, die Eröffnungsrede zu halten, und erwarteten eine Wiederholung des Auftritts von Hilbert im Jahr 1900 in Paris. Wie Hilbert eine Liste ungelöster Probleme vorgelegt hatte, um der Mathematik in der ersten Hälfte des zwanzigsten Jahrhunderts die Richtung vorzugeben, sollte von Neumann dies für die zweite Hälfte des Jahrhunderts tun. Der Titel seines Vortrags wurde im Kongressprogramm angekündigt. Er lautete: „Unsolved Problems in Mathematics. Address by Invitation of the Organizing Committee“ („Ungelöste Probleme der Mathematik. Ansprache auf Einladung des Organisationskomitees“). Nach dem Kongress wurde ein vollständiger Tagungsbericht veröffentlicht, in dem die Texte aller Vorträge außer diesem abgedruckt sind. Er enthält eine leere Seite mit von Neumanns Namen und dem Titel seines Vortrags. Darunter steht: „Von diesem Beitrag war kein Manuskript verfügbar."

Was war passiert? Ich weiß es, weil ich am Donnerstag, dem 2. September 1954 um 15 Uhr im Concertgebouw im Publikum war. Der Saal war bis auf den letzten Platz mit Mathematikern gefüllt, die einen glänzenden Vortrag erwarteten, der eines solchen historischen Augenblicks würdig ist. Doch die Rede war eine riesige Enttäuschung. Wahrscheinlich hatte von Neumann bereits einige Jahre zuvor zugesagt, einen Vortrag über ungelöste Probleme zu halten, und ihn dann vergessen. Da er mit so vielen anderen Dingen befasst war, hatte er versäumt, den Vortrag vorzubereiten. Als ihm dann im letzten Moment einfiel, dass er nach Amsterdam reisen und etwas über Mathematik sagen musste, holte er eine alte Rede aus den 193oer-Jahren wieder aus der Schublade und klopfte den Staub ab. Darin ging es um Operatorenringe, ein Thema, das in den 193oer-Jahren neu und aktuell gewesen war. Kein Wort über ungelöste Probleme. Kein Wort über die Zukunft. Kein Wort über Computer, 
das Thema, von dem wir wussten, dass es von Neumann besonders am Herzen lag. Er hätte wenigstens etwas Neues und Aufregendes über Computer erzählen können. Das Publikum im Konzertsaal wurde ungeduldig. Jemand sagte laut genug, um es im gesamten Saal zu hören: „Aufgewärmte Suppe“. 1954 konnten die allermeisten Mathematiker genügend Deutsch, um diesen Spruch zu verstehen. Von Neumann war äußerst betreten, beendete rasch seinen Vortrag und verließ den Saal, ohne auf Fragen zu warten.

\section{Schwaches Chaos}

Hätte von Neumann bei seinem Vortrag in Amsterdam etwas über das Chaos gewusst, wäre eines der ungelösten Probleme, über die er hätte sprechen können, das schwache Chaos gewesen. Auch fünfzig Jahre später ist die Frage des schwachen Chaos immer noch nicht gelöst. Die Schwierigkeit besteht darin $\mathrm{zu}$ verstehen, warum chaotische Bewegungen oft begrenzt bleiben und keine radikale Instabilität bewirken. Ein gutes Beispiel für schwaches Chaos sind die Umlaufbewegungen von Planeten und Satelliten im Sonnensystem. Erst kürzlich fand man heraus, dass diese Bewegungen chaotisch sind. Das war eine überraschende Entdeckung, die das traditionelle Bild des Sonnensystems als Musterbeispiel für eine geordnete, stabile Bewegung erschütterte. Vor zweihundert Jahren glaubte der Mathematiker Laplace bewiesen zu haben, dass das Sonnensystem stabil ist. Nun stellt sich heraus, dass Laplace falsch lag. Exakte numerische Integrationen der Umlaufbahnen zeigen sehr klar, dass benachbarte Umlaufbahnen exponentiell auseinanderdriften. Das Chaos ist in der Welt der klassischen Dynamik anscheinend fast allgegenwärtig. Bevor genaue Langzeitintegrationen durchgeführt wurden, vermutete man im Sonnensystem deshalb kein chaotisches Verhalten, weil dieses Chaos nur schwach ist. Schwaches Chaos bedeutet, dass benachbarte Bewegungsbahnen exponentiell auseinanderlaufen, aber nie besonders weit divergieren. Die Divergenz wächst zunächst exponentiell an, bleibt dann jedoch begrenzt. Weil das Chaos der Planetenbewegungen nur schwach ist, kann das Sonnensystem vier Milliarden Jahre fortbestehen. Auch wenn ihre Bewegungen chaotisch sind, entfernen sich die Planeten niemals weit von ihren gewöhnlichen Positionen, und das System insgesamt fliegt nicht auseinander. Obwohl in ihm das Chaos vorherrscht, ist die Laplace'sche Vorstellung vom Sonnensystem als perfektem Uhrwerk nicht weit von der Wahrheit entfernt.

Die gleichen Phänomene des schwachen Chaos lassen sich auch in der Meteorologie beobachten. Zwar ist das Wetter in New Jersey ausgesprochen chaotisch, doch dieses Chaos hat feste Grenzen. Ob die Sommer und Winter mild oder streng werden, ist nicht vorherzusehen, wir können jedoch zuverlässig voraussagen, dass die Temperatur niemals auf 45 Grad Celsius ansteigen oder auf minus 30 Grad fallen wird, Extreme, die in Indien oder Minnesota häufig übertroffen werden. Es gibt keinen physikalischen Erhaltungssatz, der ausschließt, dass die Temperaturen in New Jersey so hoch ansteigen wie in Indien oder dass sie in New Jersey so tief fallen wie in Minnesota. Dass das Chaos nur schwach ist, hat für den langfristigen Fortbestand des Lebens auf diesem Planeten grundlegende Bedeutung. Das schwache Chaos beschert uns bei den Wetterbedingungen eine reizvolle Abwechslung und schützt uns gleichzeitig vor Schwankungen, die so stark wären, dass sie unsere Existenz bedrohten. Das Chaos bleibt aus für uns unverständlichen Gründen glücklicherweise schwach. Auch das ist ein ungelöstes Problem, das die jungen Frösche im Publikum mit nach Hause nehmen sollten. Ich möchte Sie dazu ermuntern, den Gründen dafür nachzugehen, dass das in den unterschiedlichsten dynamischen Systemen beobachtete Chaos normalerweise schwach ist.

Das Thema Chaos zeichnet sich durch einen Überfluss an quantitativen Daten, einen unendlichen Fundus an schönen Bildern und das Fehlen rigoroser Sätze aus. Rigorose Sätze sind der beste Weg, um einem Thema intellektuelle Tiefe und Genauigkeit zu verleihen. Solange man keine rigorosen Sätze beweisen kann, versteht man die Bedeutung seiner Ansätze nicht in vollem Umfang. Auf dem Gebiet des Chaos kenne ich nur einen rigorosen Satz, der 1975 von Tien-Yien Li und Jim Yorke bewiesen und in einem kurzen Beitrag mit dem Titel „Period Three Implies Chaos“ („Periode drei bedeutet Chaos") [4] veröffentlicht wurde. Die Abhandlung von Li und Yorke ist ein unvergängliches Juwel der mathematischen Literatur. Ihr Satz betrifft nichtlineare Abbildungen eines Intervalls auf sich selbst. Die aufeinanderfolgenden Positionen eines Punktes bei Wiederholung der Abbildung können als die Umlaufbahn eines klassischen Teilchens aufgefasst werden. Eine Umlaufbahn hat Periode $N$, wenn der Punkt nach $N$ Abbildungen an seine ursprüngliche Position zurückkehrt. Eine Umlaufbahn wird in diesem Zusammenhang als chaotisch definiert, wenn sie von allen periodischen Umlaufbahnen abweicht. Der Satz besagt, dass es dann, wenn eine einzige Umlaufbahn mit Periode drei existiert, auch chaotische Umlaufbahnen gibt. Der Beweis ist einfach und kurz. Meiner Meinung nach bringen dieser Satz und sein Beweis mehr Licht in die grundlegende Natur des Chaos als tausend wunderschöne Bilder. Der Satz erklärt, warum in der Welt das Chaos vorherrscht. Er erklärt jedoch nicht, warum das Chaos so oft schwach ist. Das ist nach wie vor eine Aufgabe für die Zukunft. Ich glaube, dass schwaches Chaos erst dann grundlegend verstanden sein wird, wenn man rigorose Sätze darüber beweisen kann.

\section{Stringtheoretiker}

Ich möchte einige Worte zur Stringtheorie sagen. Nur einige Worte, weil ich sehr wenig über die Stringtheorie weiß. Ich habe mir nie die Mühe gemacht, mich in dieses Thema einzuarbeiten oder selbst darüber zu forschen. Aber zu Hause am Institute for Advanced Study in Princeton bin ich von Stringtheoretikern umgeben, und gelegentlich verfolge ich ihre Diskussionen. Manchmal verstehe ich auch etwas von dem, was sie sagen. Drei Dinge stehen fest. Erstens: Was sie machen, ist Mathematik auf höchstem Niveau. Die führenden Vertreter der reinen Mathematik, Leute wie Michael Atiyah und Isadore Singer, lieben die Stringtheorie. Sie hat ein völlig neues Gebiet der Mathematik mit 


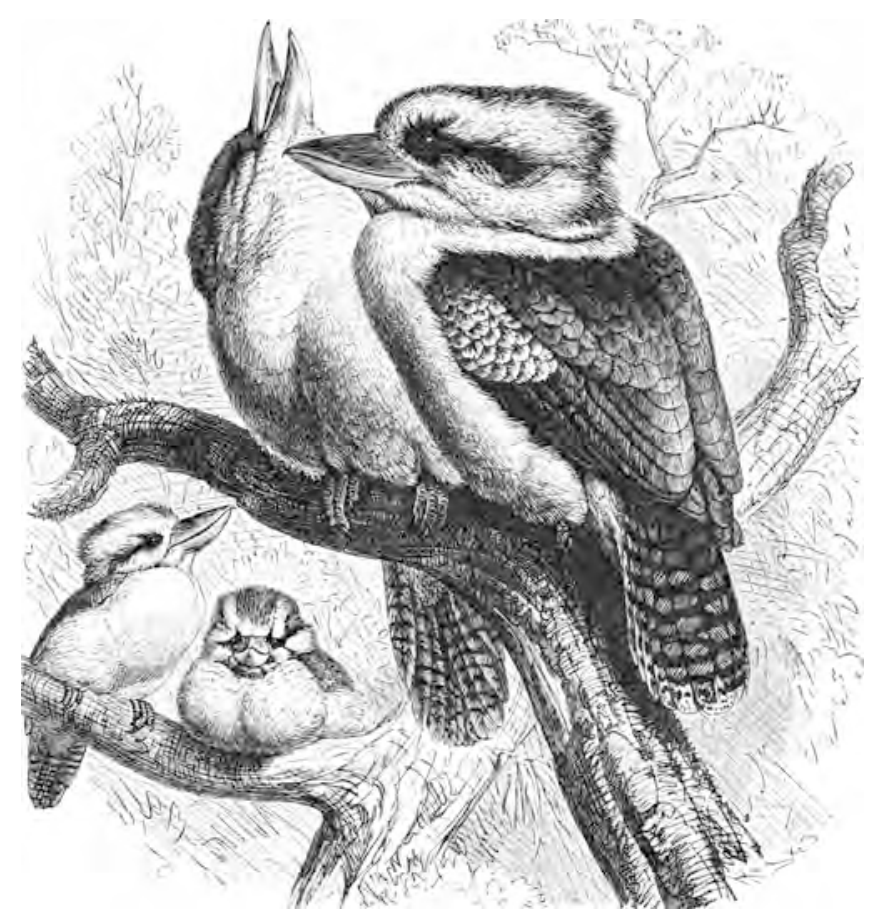

neuen Konzepten und neuen Problemen erschlossen. Besonders hervorzuheben ist, dass sie den Mathematikern neue Methoden an die Hand gegeben hat, um alte Probleme zu lösen, die bis dahin unlösbar waren. Zweitens verstehen sich die Stringtheoretiker eher als Physiker denn als Mathematiker. Sie gehen davon aus, dass ihre Theorie etwas in der physischen Welt Reales beschreibt. Und drittens gibt es bislang keinen Beweis dafür, dass diese Theorie für die Physik relevant ist. Sie ist noch nicht experimentell überprüfbar. Unabhängig vom Rest der Physik verbleibt sie in ihrer eigenen Welt. Stringtheoretiker unternehmen enorme Anstrengungen, um Schlussfolgerungen aus der Theorie zu ziehen, die in der realen Welt überprüfbar sein könnten, bislang ohne Erfolg.

Meine Kollegen Ed Witten, Juan Maldacena und andere Begründer der Stringtheorie sind Vögel, die in großer Höhe fliegen und eine großartige Sicht auf ferne Bergketten haben. Die Tausenden bescheideneren Praktiker der Stringtheorie an Universitäten auf der ganzen Welt sind Frösche und erforschen die feinen Details der mathematischen Strukturen, die die Vögel zuvor am Horizont erblickten. Meine Bedenken gegenüber der Stringtheorie sind eher soziologischer als wissenschaftlicher Art. Es ist eine ehrenvolle Angelegenheit, zu den ersten tausend Stringtheoretikern zu gehören, die neue Verbindungen herstellen und neuen Methoden den Weg bereiten. Weniger ruhmreich ist es, zu den zweiten Tausend oder zu den zehnten Tausend zu gehören. Inzwischen gibt es über die ganze Welt verteilt etwa zehntausend Stringtheoretiker. Das ist für das zehnte Tausend und vielleicht auch schon für das zweite eine gefährliche Situation. Denn der Zeitgeschmack kann sich jederzeit ändern und die Stringtheorie aus der Mode kommen. Dann verlieren womöglich neuntausend Stringtheoretiker ihren Arbeitsplatz. Sie sind in einem sehr begrenzten Forschungsgebiet ausge- bildet und in anderen Bereichen der Wissenschaft vielleicht nicht einsetzbar.

Warum aber zieht die Stringtheorie so viele junge Leute an? Diese Anziehungskraft ist zum Teil intellektueller Natur. Die Stringtheorie ist kühn und mathematisch elegant. Doch ihr Reiz ist gleichermaßen soziologischer Natur. Die Stringtheorie ist attraktiv, weil sie Arbeitsplätze bietet. Und warum werden im Bereich der Stringtheorie so viele Arbeitsplätze angeboten? Weil die Stringtheorie billig ist. Wenn man als Leiter einer Physikabteilung an einem abgelegenen Ort nicht viel Geld hat, kann man es sich nicht leisten, ein modernes Labor für Experimentalphysik zu bauen, aber man kann es sich leisten, ein paar Stringtheoretiker anzustellen. Man schafft also einige Arbeitsplätze im Bereich der Stringtheorie, und schon hat man eine Abteilung für moderne Physik. Die Verlockung, solche Stellen anzubieten, ist für den Institutsleiter groß, und ebenso groß ist die Verlockung für junge Leute, sie anzunehmen. Dies ist sowohl für die jungen Menschen als auch für die Zukunft der Wissenschaft eine gefährliche Situation. Ich möchte damit nicht sagen, dass wir junge Wissenschaftler davon abhalten sollten, sich mit der Stringtheorie zu beschäftigen, wenn sie sie interessant finden. Ich meine nur, dass wir ihnen Alternativen anbieten sollten, damit sie nicht aus wirtschaftlicher Notwendigkeit in die Stringtheorie hineingedrängt werden.

Abschließend möchte ich Ihnen sagen, wie ich persönlich die Zukunft der Stringtheorie einschätze. Wahrscheinlich ist meine Vermutung falsch. Ich bilde mir nicht ein, die Zukunft vorhersagen zu können. Ich gebe Ihnen meine Einschätzung nur als Anregung, darüber nachzudenken. Ich halte es für unwahrscheinlich, dass sich die Stringtheorie als in jeder Hinsicht erfolgreich oder als vollkommen unbrauchbar erweisen wird. Mit „in jeder Hinsicht erfolgreich“ meine ich, dass sie eine vollständige physikalische Theo- 
rie ist, die Teilchen und ihre Wechselwirkungen in allen Einzelheiten beschreibt. Mit „vollkommen unbrauchbar“ meine ich, dass sie nur ein schönes Stück reiner Mathematik bleibt. Meine Vermutung ist, dass die Stringtheorie irgendwo zwischen Erfolg in jeder Hinsicht und Misserfolg enden wird. Ich schätze, dass es ihr wie der Theorie der Lie-Gruppen ergehen wird, die Sophus Lie im neunzehnten Jahrhundert als mathematischen Rahmen für die klassische Physik aufgestellt hat. Innerhalb der klassischen Physik blieben Lie-Gruppen ein Misserfolg. Sie waren eine Lösung auf der Suche nach einem Problem. Doch dann veränderte die Quantenrevolution fünfzig Jahre später die Physik, und die Lie-Algebren fanden ihren Platz. Sie wurden der Schlüssel für das Verständnis der zentralen Rolle von Symmetrien in der Welt der Quanten. Ich erwarte, dass es in fünfzig oder hundert Jahren eine weitere Revolution in der Physik geben wird, die neue Konzepte mit sich bringt, von denen wir heute noch keine Vorstellung haben, und diese neuen Konzepte werden der Stringtheorie eine neue Bedeutung geben. Die Stringtheorie wird plötzlich den rechten Ort im Universum finden und überprüfbare Aussagen über die reale Welt machen. Ich möchte Sie darauf hinweisen, dass diese Einschätzung der Zukunft wahrscheinlich falsch ist. Das Gute an ihr ist, dass sie falsifizierbar ist, was Karl Popper zufolge das Qualitätsmerkmal einer wissenschaftlichen Aussage ist. Sie könnte schon morgen durch eine Entdeckung aus dem Großen Hadronen-Speicherring in Genf widerlegt werden.

\section{Noch einmal Manin}

Zum Ende dieses Vortrags möchte ich noch einmal auf Yuri Manin und sein Buch Mathematics as Metaphor zurückkommen. In dem Buch geht es vor allem um Mathematik. Es wird den westlichen Leser überraschen, dass Manin mit der- selben Eloquenz über weitere Themen wie das kollektive Unbewusste, den Ursprung der menschlichen Sprache, die Psychologie des Autismus und die Rolle des Schwindlers in der Mythologie vieler Kulturen schreibt. Für seine russischen Landsleute sind solche vielseitigen Interessen und Sachkenntnisse keine große Überraschung. Russische Intellektuelle pflegen die stolze Tradition der alten russischen Intelligenz, nach der Wissenschaftler und Dichter, Künstler und Musiker einer einzigen Gemeinschaft angehören. Sie sind auch heute noch eine Gruppe von Idealisten wie in den Stücken von Tschechow, die durch ihre Abwendung von einer abergläubischen Gesellschaft und einer unberechenbaren Regierung miteinander verbunden sind. In Russland sprechen Mathematiker, Komponisten und Filmproduzenten miteinander, gehen an Winterabenden gemeinsam im Schnee spazieren, sitzen bei einer Flasche Wein zusammen und tauschen ihre Gedanken aus.

Manin ist ein Vogel, dessen Blick weit über das Territorium der Mathematik hinaus in die ausgedehntere Landschaft menschlicher Kultur reicht. Eines seiner Hobbys ist die von dem Schweizer Psychologen Carl Jung entwickelte Theorie der Archetypen. Ein Archetyp ist Jung zufolge ein geistiges Bild, das in einem uns allen gemeinsamen kollektiven Unbewussten wurzelt. Die intensiven Gefühle, die Archetypen mit sich bringen, sind Überbleibsel verlorener Erinnerungen an gemeinsame Freuden und Leiden. Für Manin ist es nicht notwendig, Jungs Theorie als wahr anzunehmen, um sie erhellend $\mathrm{zu}$ finden.

Vor mehr als dreißig Jahren machte die Sängerin Monique Morelli eine Aufnahme von Liedern mit Texten von Pierre MacOrlan. Eines der Lieder ist La Ville Morte, die tote Stadt, mit einer auf Morellis tiefen Alt abgestimmten ergreifenden Melodie, einem Akkordeon, das einen Kontrapunkt zur Stimme bildet, und Sprachbildern von außergewöhnlicher Intensität [youtu.be/yYFopXZRGms, Anm. d. Red.]. Auf Papier gedruckt sind die Worte nichts Besonderes:

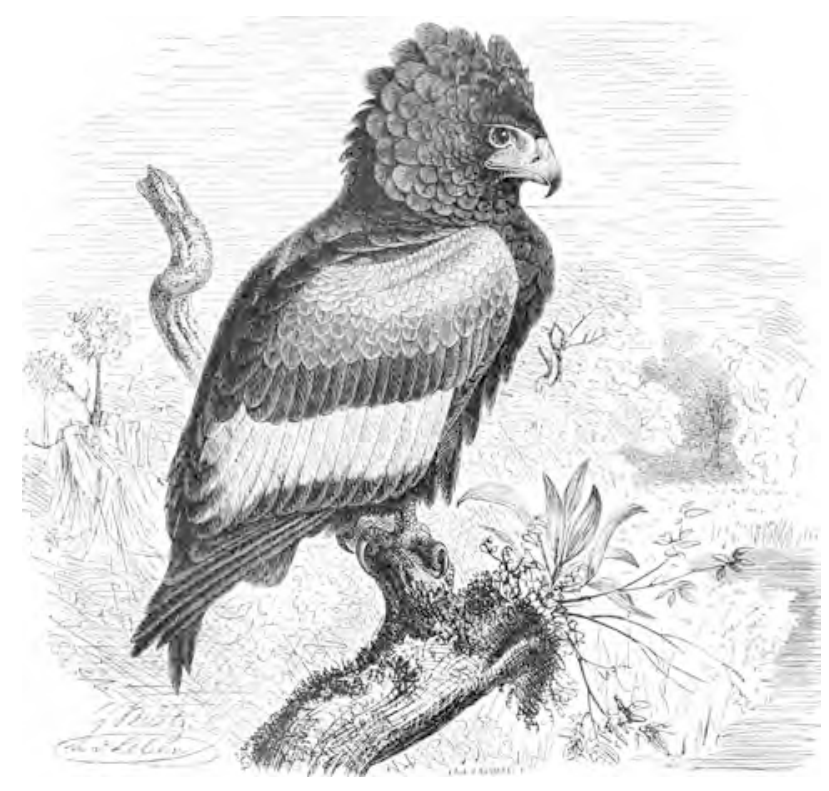


En pénétrant dans la ville morte, Je tenait Margot par le main... Nous marchions de la nécropole, Les pieds brisés et sans parole, Devant ces portes sans cadole, Devant ces trous indéfinis, Devant ces portes sans parole Et ces poubelles pleines de cris.

Als wir in die tote Stadt kamen, hielt ich Margot an der Hand ... Wir gingen vom Gräberfeld auf zerschundenen Füßen ohne ein Wort vorbei an diesen Toren ohne Haus, vorbei an diesen unbestimmten Löchern, vorbei an diesen Toren ohne ein Wort und an diesen Kehrichttonnen voll Geschrei.

Ich kann dieses Lied nicht ohne maßlos übertriebene Gefühlsregungen hören. Oft frage ich mich, warum die schlichten Worte des Liedes anscheinend eine tiefe, unbewusste Ebene der Erinnerung berühren, als sprächen die Seelen der Verstorbenen durch Morellis Musik. Und in Manins Buch finde ich nun ganz unverhofft eine Antwort auf meine Frage. In dem Kapitel „The Empty City Archetype“ („Der Archetyp der leeren Stadt") beschreibt Manin, wie der Archetyp der toten Stadt in den Werken der Architektur, der Literatur, der Kunst und des Films von der Antike bis zur Moderne immer wieder auftaucht, seit sich Menschen in Städten zusammenfanden, seit sich andere Menschen in Armeen zusammenfanden, um die Städte zu verwüsten und zu zerstören. In MacOrlans Lied spricht ein alter Soldat zu uns, der vor langer Zeit einer Besatzungsarmee angehört hatte. Nachdem er mit seiner Frau durch den Staub und die Asche der toten Stadt gegangen ist, vernimmt er noch einmal:

Chansons de charme d'un clairon

Qui fleurissait une heure lointaine

Dans un rêve de garnison.

Die magischen Rufe eines Horns, das im Traum eines alten Soldaten eine Stunde lang zum Leben erwachte.

MacOrlans Worte und Morellis Stimme scheinen einen Traum unseres kollektiven Unbewussten zum Leben zu erwecken, den Traum eines alten Soldaten, der durch eine tote Stadt wandert. Der Gedanke eines kollektiven Unbewussten mag ebenso mythisch sein wie der Gedanke einer toten Stadt. Manins Kapitel beschreibt das dezente Licht, das diese beiden womöglich mythischen Vorstellungen aufeinander werfen. Er beschreibt das kollektive Unbewusste als irrationale Kraft, die uns machtvoll in Richtung Tod und Zerstörung zieht. Der Archetyp der toten Stadt ist eine Essenz aus den Leiden hunderter realer Städte, die seit der Erfindung von Städten und marodierenden Armeen zerstört wurden. Unsere einzige Möglichkeit, dem Irrsinn des kollektiven Unbewussten zu entkommen, ist ein gesundes kollektives Bewusstsein, das auf Hoffnung und Vernunft beruht. Die große Aufgabe, vor der die heutige Zivilisation steht, ist es, ein solches kollektives Bewusstsein zu schaffen.

Aus dem Amerikanischen von Sylvia Zirden

\section{Literatur}

[1] M. J. Bertin u. a., Pisot and Salem Numbers. Birkhäuser Verlag, Basel, 1992.

[2] M. L. Cartwright und J. E. Littlewood, On nonlinear differential equations of the second order, I. Jour. London Math. Soc. 20 (1945), 180-189.

[3] Freeman Dyson, Prof. Hermann Weyl, For. Mem. R. S. Nature 177 (1956), 457-458.

[4] Tien-Yien Li und James A. Yorke, Period three implies chaos. Amer. Math. Monthly 82 (1975), 985-992.

[5] Yuri I. Manin, Mathematics as Metaphor: Selected Essays. American Mathematical Society, Providence, Rhode Island, 2007. [Russische Ausgabe: Matematika kak Metafora, Moskau, Izdatyelstvo MTsNMO, 2008.]

[6] Andrew M. Odlyzko, Primes, quantum chaos and computers. In Number Theory, Proceedings of a Symposium. National Research Council, Washington DC, 1990, S. 35-46.

[7] Hermann Weyl, Gravitation und Elektrizität. Sitz. König. Preuss. Akad. Wiss. 26 (1918), 465--48o.

[8] —, Elektron und Gravitation. Zeits. Phys. 56 (1929), 350-352.

[9] —, Selecta. Birkhäuser Verlag, Basel, 1956, S. 192.

[10] Chen Ning Yang, Integral formalism for gauge fields. Phys. Rev. Letters 33 (1974), 445--447.

[11] Chen Ning Yang und Robert L. Mills, Conservation of isotopic spin and isotopic gauge invariance. Phys. Rev. 96 (1954), 191-195.

[12] —, Hermann Weyl's contribution to physics. In Hermann Weyl, $1885-1985$, hrsg. v. K. Chandrasekharan. Springer-Verlag, Berlin, 1986, S. 19 .

"Birds and Frogs" ist eine schriftliche Version von Freeman Dysons (nicht gehaltener) AMS Einstein Lecture 20o8, zuerst erschienen in den Notices of the American Mathematical Society 56, no. 2 (2009), 212-223. (C) 2009 American Mathematical Society. Mit freundlicher Genehmigung der AMS.

Abbildungen (Public Domain) aus Brehms Tierleben. Allgemeine Kunde des Thierreichs, Bibliographisches Institut, Leipzig und Wien S. 30: Bd. 4, Tafel XVI, [1912] 1920 · . 31: Bd. 7, S. 672, $1900 \cdot$ S. 32: Bd. 4, S. 263, 1913 · S. 34: Bd. 1, S. 255,1912

S. 35: Bd. 1, S. 302, 1912 .S. 37: Bd. 3, S. 208, 1911 .S. 39: Bd. 1, S. 249, 1912 . S. 41: Bd. 3, S. 67, $1900 \cdot /$ S. 42: Bd. 3, S. 312, 1900 